\title{
Detecting Malicious Network Traffic Using Inverse Distributions of Packet Contents
}

\author{
Vijay Karamcheti, Davi Geiger, Zvi Kedem \\ Courant Institute of Mathematical Sciences \\ New York University \\ \{vijayk,geiger,kedem\}@cs.nyu.edu
}

\author{
S. Muthukrishnan \\ Department of Computer Science \\ Rutgers University \\ muthu@cs.rutgers.edu
}

\begin{abstract}
We study the problem of detecting malicious IP traffic in the network early, by analyzing the contents of packets. Existing systems look at packet contents as a bag of substrings and study characteristics of its base distribution $B$ where $B(i)$ is the frequency of substring $i$.

We propose studying the inverse distribution $I$ where $I(f)$ is the number of substrings that appear with frequency $f$. As we show using a detailed case study, the inverse distribution shows the emergence of malicious traffic very clearly not only in its "static" collection of bumps, but also in its nascent "dynamic" state when the phenomenon manifests itself only as a distortion of the inverse distribution envelope. We describe our probabilistic analysis of the inverse distribution in terms of Gaussian mixtures, our preliminary solution for discovering these bumps automatically. Finally, we briefly discuss challenges in analyzing the inverse distribution of IP contents and its applications.
\end{abstract}

\section{Categories and Subject Descriptors}

D.4.6 [Security and Protection]: Invasive software

\section{General Terms}

Security

\section{Keywords}

worms, inverse distribution, content analysis

\section{INTRODUCTION}

To cope with increasingly sophisticated network attacks, a growing number of network security tools inspect the contents (as opposed to just headers) of network packets, either individually or at the level of flows. For the most part, such inspection is restricted to matching packet contents against a pre-established set of signature patterns. However, researchers $[8,15,9,13]$ have recently started advocating more general analysis of traffic contents to try and discern signals indicative of malicious network activity.

Permission to make digital or hard copies of all or part of this work for personal or classroom use is granted without fee provided that copies are not made or distributed for profit or commercial advantage and that copies bear this notice and the full citation on the first page. To copy otherwise, to republish, to post on servers or to redistribute to lists, requires prior specific permission and/or a fee.

SIGCOMM'05 Workshops, August 22-26, 2005, Philadelphia, PA, USA.

Copyright 2005 ACM 1-59593-026-4/05/0008 ...\$5.00.
Our work follows this trend, investigating whether statistical analysis of the contents of packets seen at one or more network elements can be used to detect the onset of a network-wide computer worm attack. Our approach complements perimeter defenses such as portscan detectors [7] and anomaly-based firewall and IDS systems by looking for the emergence of new patterns in packet contents. Thus, it can be used to detect attacks that target buggy but otherwise functional network services running on vulnerable hosts; depending on the sophistication of the worm code, the defenses above may or may not be able to detect such attacks.

The primary intuition underlying our approach is that an ongoing worm propagation should manifest itself in the presence of higher than expected byte-level similarity among network packets: this similarity arises because of the unchanging portions of the worm packet payload, something expected to be present even in polymorphic or obfuscated worms (albeit spread out over the length of the packet). ${ }^{1}$ A similar intuition has been explored by other researchers. The EarlyBird system [15] looks for frequently occuring substrings in packet contents as indicators of potentially malicious content. A similar idea can also be found in the Autograph [9] and Polygraph [13] systems; these systems reason about the frequency with which patterns of substrings appear to generate compact and discriminating signatures for a collection of packets classified by an external entity as being malicious.

All three systems represent packet contents as a bag of substrings (of either a fixed length [15], or a dynamic packet content-based length $[9,13])$. The analysis looks at the characteristics of the resultant base distribution, $B(i)$, which tracks the frequency with which a specific substring $i$ appears in a collection of packets.

In contrast, our work analyzes the characteristics of the inverse distribution, $I(f)$, which tracks for a given frequency $f$, the number of substrings that appear with that frequency. As we show in Section 2, as compared to the base distribution, the inverse distribution appears to permit earlier, more discriminating detection of the emergence of new sources of content similarity, which in turn serve as indicators of malicious traffic. In fact, the presence of a worm was detected with fewer than 50 of the worm packets having appeared in a stream of 20,000 packets. Section 3 describes our preliminary approaches for analyzing inverse distributions to detect and track content similarity "features". These approaches rely upon a probabilistic model for the shape of the distribution, and emphasize early detection of an attack, with a very small number of worm packet instances and with as few false positives as possible. Section 4 discusses incorporation of inverse-distribution based analyses into network security applications.

\footnotetext{
${ }^{1}$ Note that this observation assumes that either the traffic is unencrypted or that our techniques are deployed in a location where traffic can be decrypted as required.
} 

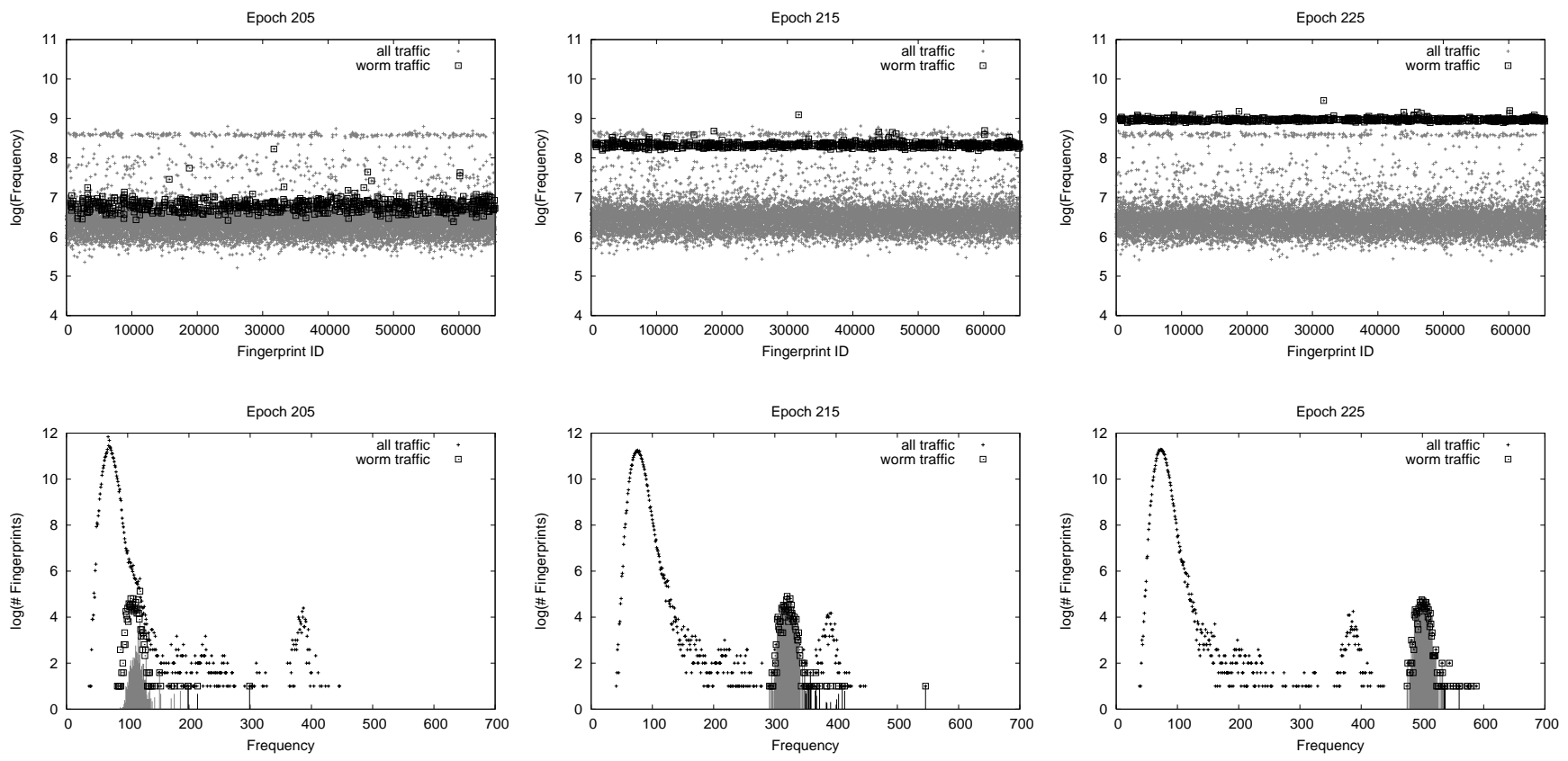

Figure 1: Base (top) and inverse (bottom) frequency distributions of fingerprints over a 20,000 packet window observed at three different time epochs: Epoch 205 corresponds to the appearance of 22 Witty worm packets, Epoch 215 to 132 packets, and Epoch 225 to 242 packets. The fingerprints appearing in all worm packets are shown using the square points. The gray bars under the inverse distribution show regions containing fingerprints that have been displaced with respect to the previous time epoch by a large value. Note that the inverse distribution shows the presence of the worm from Epoch 205 itself.

\section{CASE STUDY}

The benefits of inverse distributions vis-a-vis base distributions are highlighted by a case study that we conducted. We captured packets on our research network (a /24 address range subnetted from a Class $\mathrm{B}$ address, with about 60 hosts active at any one time) over a 15-minute period. The packet trace, containing roughly 75,000 incoming and outgoing UDP and TCP packets, corresponded to a variety of applications including web server requests and responses, interactions with mail servers using POP and IMAP, file server access, and IM and chat traffic.

Into this trace, we synthetically injected UDP packets corresponding to two well-known worms: the SQL Slammer worm and the W32.Witty worm. Packets containing the SQL Slammer worm appeared throughout the trace at a relatively low frequency (1 worm packet for every 64 data packets) and correspond to the presence of known worms in network traffic; typically, such worm packets continue to propagate long after the worm has been detected and counteracted. The Witty worm packets correspond to a new, as yet unknown worm, and start appearing 20,000 packets into the trace at a higher rate: 1 worm packet for every 8 data packets. Our objective was to examine whether any signals indicative of this new worm could be discerned by analyzing the base and inverse distributions of packet contents and whether one distribution presented any advantages over the other.

To build these distributions, we represent packet contents as in earlier work by a bag of "shingles" [1] denoting different substrings. We derive a smaller $p$-bit "fingerprint" for each overlapping $k$-byte segment ("shingle") of the packet body, one for each byte boundary. Each packet is represented by the set of fingerprints it contains, and the contents of all packets seen by a router is compactly captured by either the base or the inverse frequency distribution of these fingerprints. Intuitively, two packets exhibiting byte-level similarity would share a set of shingles, while two dissimilar packets would not. Thus, as the system sees an increasing number of worm packets, we would expect the fingerprints derived from such packets to appear with frequencies higher than the other fingerprints. These "frequency gaps" are manifested differently in the base and inverse distributions, leading to differences in how suitable a particular distribution might be in identifying packets that are possibly malicious (in the sense that they contain highfrequency fingerprints, hence the corresponding shingles).

Fig. 1 shows the base (top) and inverse (bottom) frequency distributions (with $k=20$ and $p=16$ ) over a 20,000 packet window; the $y$-axis in both plots uses the log scale. The plots correspond to three time instants: (Epoch 205) where 22 packets of the Witty worm have been seen, (Epoch 215) with 132 worm packets, and (Epoch 225) with 242 worm packets.

Signal in the base distribution. The base distribution plots clearly show the movement of the fingerprints shared by all worm packets (shown using square points), corresponding to the frequency gap described above. Although the movement is present throughout, it is only at Epoch 225 that the highest frequency fingerprints in the base distribution do in fact correspond to the worm.

Systems like EarlyBird, Autograph, and Polygraph distinguish among the fingerprints based on a frequency threshold: fingerprints that appear more frequently than this threshold are tagged as potentially malicious, and those below are not. Such approaches necessarily entail a tradeoff between early detection of new worm traffic and the likelihood of false positives; the former argues for lower frequency thresholds while the latter requires higher thresholds. Even with 40-byte substrings, only a very small number of which can appear over any reasonable observation window, EarlyBird suffers 
from false positives and requires a manually created "whitelist" of good substrings known to occur frequently.

To avoid false positives without using whitelists, one would need to wait for a time instant where the frequency of the worm fingerprints exceeds that of previously known sources of content similarity (the SQL Slammer worm in this case). Not only does this delay detection to Epoch 225 in our case, but also note that whether or not a new worm's fingerprints ever correspond to the most frequently occuring set depends upon the relative rate with which the worm packets appear during the time window captured by the distribution. In particular, if the rate of Witty worm packets remains below that of the Slammer worm, detection mechanisms that analyze the most prevalent fingerprints will not yield good results. Stealthy worm attacks are particularly prone to this phenomenon: their relative rate may never exceed the threshold required for sufficiently discriminating detection.

Signal in the inverse distribution. In contrast, the inverse distribution plots in the bottom half of Fig. 1 appear not to suffer from these problems. Some additional explanation is necessary about the nature of these plots. The envelope made up from one or more "bumps" corresponds to the inverse distribution $I(f)$, which tracks for a given frequency $f$, the number of fingerprints that appear with that frequency. The contribution of the worm fingerprints to this count at each column is shown using the square points. Finally, the gray shaded regions under the inverse distribution curve show contributions from those fingerprints that have incurred displacements from the previous time epoch of more than a certain threshold. Informally, these regions show the portion of the frequency spectrum occupied by fast-moving fingerprints.

The inverse distribution plots show the emergence of the new worm packets in a clearer, unambiguous, and rate-insensitive fashion. At Epoch 215, the new worm packets are discernible because of the creation of a new "bump" between the two originally present at Epoch 205 and earlier. One might argue that a similar feature can also be detected from the base distribution at the same time instant, particularly if we look for gaps in the frequency distribution instead of just focusing on the highest frequency fingerprints. Note however that unlike in the base distribution, in the inverse distribution at Epoch 205, the worm is already noticeable after only 22 packets: here, their presence distorts the first bump in a way consistent with a new bump breaking away from the original one. Moreover, for fast spreading worms, tracking the various fingerprint displacements (the gray regions in the plots) may in fact yield a more responsive detector that raises an alert even before any perceptible distortion is observed in the inverse distribution envelope.

Although the case study looks at a small amount of network data and synthetic worm propagation behaviors, it highlights the potential of using inverse distributions: the analysis of the structure and location of the bumps in this distribution appears to provide a highly discriminating, responsive mechanism for characterizing different sources of content similarity.

\section{TRACKING FEATURES INDICATING CONTENT SIMILARITY}

We consider a network element that at time $t$ has access to a history of base distributions $B_{t-m+1}, B_{t-m+2}, \ldots, B_{t}$ and a history of inverse distributions $I_{t-k+1}, I_{t-k+2}, \ldots, I_{t}$ ( $k$ can be larger than $m$ because inverse distributions are typically more compact). The distributions store information about packet fingerprints observed over a sliding fixed-size time window: since packets seen in the past cannot be retained, each distribution is necessarily approximate (we use exponential weighting to "forget" packets at the trail- ing edge of the window). Comparing the frequency value of a fingerprint across the sequence of base distributions allows us to compute its displacement and thereby construct the gray regions shown in Fig. 1. We want to (1) characterize the bumps in the inverse distribution (in terms of their number and location, the set of fingerprints that make up the bumps, etc.); and (2) track these bumps over time as they appear, move, and disappear.

Below, we sketch several approaches we have been pursuing towards this objective; we have focused so far on detection ability and robustness concerns rather than implementation efficiency. The approaches rely on a probabilistic model for the shape of possible inverse distributions.

\subsection{Modeling the Inverse Distribution}

We start by modeling the inverse distribution for background traffic in the absence of any packet groups exhibiting content similarity, and then extend it to account for such groups.

Background traffic. Each packet's content is represented by a set of fingerprints. Our basic model assumes that the fingerprints are produced by a uniform distribution. With $p$-bit fingerprints, there are at most $N_{\mathrm{F}}=2^{p}$ different fingerprints. For ideal fingerprinting mechanisms, a random shingle is hashed to fingerprint $i$ with probability $p(i)=p=1 / N_{\mathrm{F}}$.

Since the probability of each fingerprint is uniform and independent of the previous ones, the probability that some particular fingerprint $i$ has appeared $k$ times in the total of $n$ fingerprints seen at the router is given by the binomial distribution:

$$
\begin{aligned}
b(k, n, p(i)) & =\left(\begin{array}{l}
n \\
k
\end{array}\right) p(i)^{k}(1-p(i))^{n} \\
& \approx \frac{(n p)^{k}}{k !} e^{-n p}=p(k, n p),
\end{aligned}
$$

where the approximation by the Poisson distribution $p(k, n p)$ is valid when, as in our case, $n$ is very large and $p(i)=p=1 / N_{\mathrm{F}}$ is small such that $\lambda=n p$ is of moderate magnitude. Thus, the expected number of fingerprints that appear $k$ times is $E_{\mathrm{F}, n}(k)=$ $N_{\mathrm{F}} p(k, n p)$, an observation confirmed with real data.

Adding a single packet group to the background. We assume that the group is made up of identical packets, and corresponds to $N_{\mathrm{G}}$ distinct fingerprints (the "group fingerprints"). Thus, when a packet from the group is observed, the inverse distribution increments by one for each of these $N_{\mathrm{G}}$ fingerprints.

If there are $n_{\mathrm{G}}$ observations of each group fingerprint and $n$ observations of background traffic fingerprints, the expected number of fingerprints that appear $k$ times is:

(i) For the $N_{\mathrm{F}}-N_{\mathrm{G}}$ fingerprints that are not present in the group packets, but exist in the background traffic, following Eq. 1,

$$
E_{\mathrm{F}-\mathrm{G}, n}(k)=\left(N_{\mathrm{F}}-N_{\mathrm{G}}\right) p(k, n p) .
$$

(ii) Fingerprints that are present in the group packet will contribute to the inverse distribution each time they are observed, say $n_{\mathrm{G}}$ times. Necessarily, $k \geq n_{\mathrm{G}}$, with $n_{\mathrm{G}}$ contributed by group packets, and $k-n_{\mathrm{G}}$ contributed by the background. There are $N_{\mathrm{G}}$ of these fingerprints, so

$$
E_{\mathrm{G}, n}(k)=N_{\mathrm{G}} p\left(k-n_{\mathrm{G}}, n p\right) \quad \text { for } \quad k \geq n_{\mathrm{G}} .
$$

Combining the two cases, $E_{\mathrm{F}, n}(k)=\left(N_{\mathrm{F}}-N_{\mathrm{G}}\right) p(k, n p)+N_{\mathrm{G}} p(k-$ $\left.n_{\mathrm{G}}, n p\right)$ when $k \geq n_{\mathrm{G}}$, and $N_{\mathrm{F}} p(k, n p)$ when $k<n_{\mathrm{G}}$. Intuitively, the resulting inverse distribution consists of a Poisson component (bump) for the background and a shifted one for the group.

Generalizing to $L>1$ groups. There may be up to $2^{L}$ bumps, 


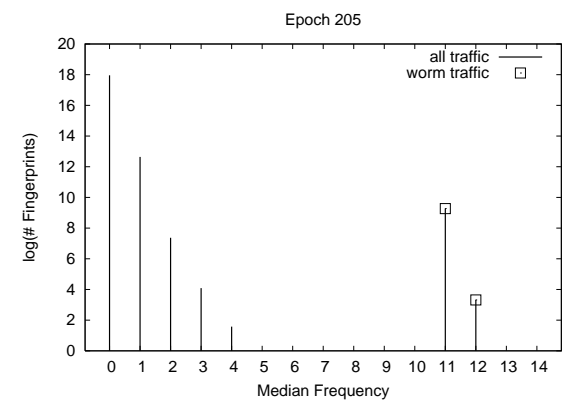

Figure 2: "Squashed" inverse distribution of fingerprints over a 100 packet window. The fingerprints appearing in Witty worm packets are shown using the square points. Note the clear separation between worm and background fingerprints.

since two or more groups may contain common fingerprints and these fingerprints will then appear more often as compared to the other fingerprints that are not common. Such combinatorics needs to be carefully modeled, but the overall structure of the model stays the same as for the single group case above: the inverse distribution is made up out of a primary background bump and some number of content similarity-induced secondary bumps.

"Squashed" inverse distributions. The above model assumed that the observation window (measured in number of fingerprints) over which the inverse distribution was being computed was much larger than the overall universe of fingerprints. Analyzing the inverse distributions that result under a different set of assumptions, specifically where the reverse is true, can also be beneficial.

Intuitively, when the fingerprint universe is significantly larger than the observation epoch, one expects a fingerprint to appear at most once in background traffic. However, fingerprints corresponding to worm packets should appear with a frequency determined by the number of worm packets observed in the observation epoch. Thus, given a sufficiently large observation epoch over which one observes multiple worm packets, and a fingerprint universe that is significantly larger, one can create inverse distributions where the fingerprints that appear only in the background traffic are essentially squashed against the y-axis.

Such squashed inverse distributions potentially permit earlier detection of malicious traffic. Fig. 2 shows the inverse distribution for the trace in Section 2 built using the median frequency value of a fingerprint over three epochs each corresponding to 100 packets. Each fingerprint was of size 18 bits resulting in a universe of size $2^{18}$; in comparison, the number of fingerprint observations in an epoch was around 30,000. The inverse distribution corresponds to the leftmost Epoch 205 in Fig. 1, but in contrast shows the presence of the worm packets more clearly.

Note that these benefits come with costs: (1) increased memory requirements for storing the distributions over a larger universe of fingerprints; and (2) increased sensitivity to benign temporary appearances of content similarity because of the compressed observation windows. In practice, one would likely want to work with both the squashed and the original inverse distributions.

\subsection{Bump Characteristics and Motion}

Guided by the models above, we analyze the base and inverse distribution data to infer information about the presence of content similarity groups in network traffic, and the set of fingerprints that define these groups. We have been exploring both direct and indi- rect approaches towards this goal. The output in either case is the identification of one or more sets of fingerprints, whose emergence, movement, and disappearance is tracked over time.

The direct approach, which we have been experimenting with in the context of squashed inverse distributions, looks for fingerprints that appear with larger than expected frequency over a number of observation epochs. These fingerprints can be identified either using a simple thresholding test on a robust statistic such as the median, or a probabilistic framework similar to sequential hypothesis testing. Fingerprints that appear with similar frequencies are grouped into the same set.

The indirect approach first detects features (bumps) in the inverse distribution and then correlates these features with the set of fingerprints that most likely resulted in their presence. For the general inverse distribution, the features correspond to the Poisson bumps. Although ideally we would like to directly estimate the Poisson parameters, to simplify the analysis we model the logarithm of the distribution as a linear combination of normal distributions (the Gaussian mixture model); each Poisson distribution is approximated by a Gaussian function $\mathscr{G}_{k}(\mu, \sigma)$ and is associated with a scale parameter $a$. Our estimation problem is one of determining these parameters for some number of Gaussian functions, say $W$, that best approximates the real data according to a mixture model:

$$
\tilde{E}_{\mathrm{F}, n}(k)=\log \left(1+E_{\mathrm{F}, n}(k)\right) \approx \sum_{i=1}^{W} a_{i} \mathscr{G}_{k}\left(\mu_{i}, \sigma_{i}\right) .
$$

The value of $W$ is unknown a priori, so it also needs to be estimated. At first glance, this problem is well-studied: there is a huge body of literature on Gaussian mixture modeling with unknown numbers of components, including integrated model selection and estimation approaches (e.g., [6]). However, we have found these approaches to be non-robust: small fluctuations in the inverse distributions across adjoining time epochs produce widely different numbers of components. Consequently, we have been pursuing a two-step procedure where we first estimate $W$ using domainspecific mechanisms, and then compute the parameters of the $W$ components using standard statistical techniques.

Estimating $W$, the number of bumps. Since a bump evolves over time in a well-known pattern modulo statistical fluctuations, we estimate $W$ by tracking the "flow" of the inverse distribution over time. The correspondence between two inverse histograms is used to obtain a displacement field, either at the detailed level of individual fingerprints (particularly those that incur large displacements), or at the level of the inverse distribution envelope. These displacements are then tracked to determine the number of bumps, $W$, and their locations.

Tracking fast-moving fingerprints. As Fig. 1 shows, the motion of the bump denoting the worm packets is accompanied by the appearance at the corresponding region of frequency values, of a group of fingerprints that have incurred large frequency changes with reference to an earlier time instant. Thus, computing for each frequency value, the number of fingerprints that have arrived there because of a large increment in their frequency, can help identify portions of the frequency range where a bump is located.

One can additionally track the patterns of how these regions themselves evolve over time to rule out potential false positives. For example, we expect a worm bump moving to the right to result in a decrease in the number of fast moving fingerprints at the previous location and an increase in the new location.

Fig. 3 shows the output of a tracker program developed using these principles. Note that as we had speculated in Section 2, the 

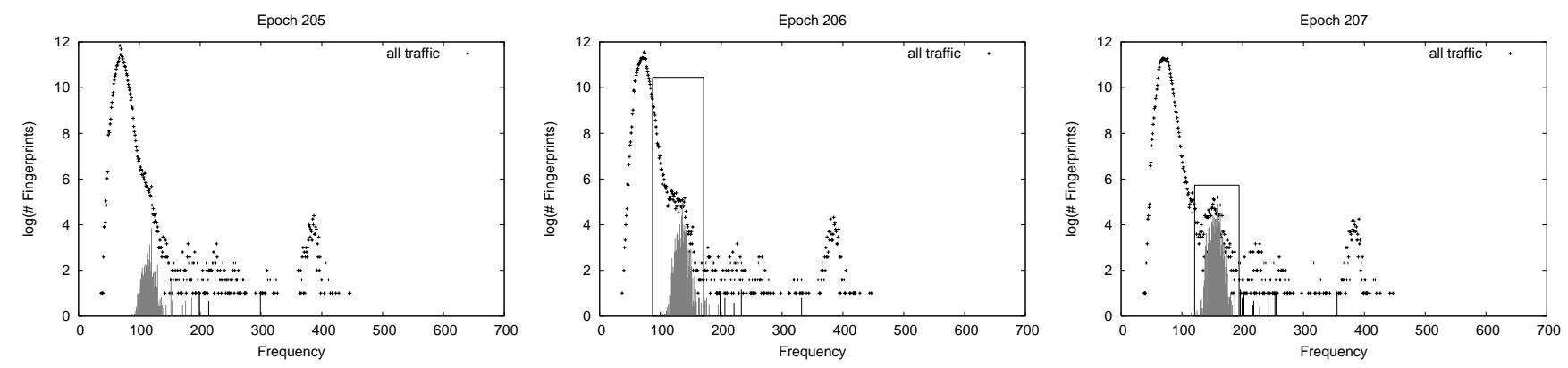

Figure 3: Tracking fast-moving fingerprints can help identify emergence of bumps indicating new sources of content similarity. For the case study described in Section 2, one can, fully automatically, detect presence of the group of Witty worm packets at Epoch 206, after only 33 worm packets have been seen.

emergence of the bump can be flagged (shown by the boxed region) with sufficient confidence well before the bump has completely separated from its "parent." In this case, we are able to flag the appearance of a new source of content similarity at Epoch 206 , after only 33 copies of the Witty worm packet were observed.

Tracking changes in the inverse distribution envelope. Direct tracking of fingerprint displacements does not pick up stealthy worms, whose propagation rate stays below a threshold. Such cases can still be detected by tracking changes in the inverse distribution envelope: rate of worm propagation can only affect how quickly these changes happen, but cannot prevent them from happening.

We use a Bayesian formulation for defining the best correspondence between two inverse distribution envelopes $I_{t}$ and $I_{t-1}$. As bumps appear or disappear they cause a portion of inverse distribution to move to the left or the right. Thus, the correspondence can be defined in terms of the displacement (in terms of frequency) seen by each point in the $I_{t-1}$ envelope for the latter to have transformed into the $I_{t}$ envelope. A particular assignment of displacement values is more probable the closer the $I_{t-1}$ envelope comes to the $I_{t}$ envelope after applying that displacement. We would also like (1) for a packet group arrival/departure to cause as few displacement changes over the prior model as possible; (2) to not skip matches for regions of the inverse distribution; and (3) to have a slight preference towards a displacement of zero. Each of these conditions can be factored into the formulation by introducing additional biases into the expression governing the probability of a particular assignment of displacement values.

A dynamic programming scheme can be used to obtain the optimal displacement value associated with each point in the $I_{t-1}$ inverse envelope (see Fig. 4). Note that both positive and negative displacement values are explored to support movements of the point to the right or left. Fig. 4 (bottom) shows the optimal displacement fields for two consecutive time epochs from a different trace: note the positive and negative steps in the displacement fields corresponding to the two non-background bumps (one moving to the right, and one to the left).

Estimating Parameters of the $W$ Gaussians. This can be done in a relatively straightforward manner once we know $W$. The parameters of the Gaussian components can be estimated, among other methods, by using a Bayesian framework involving maximum likelihood and the Expectation Maximization (EM) algorithm [4]. The initial guesses of the $a$ and $\mu$ parameters are based on the results of the change detection procedures above. Additional domain knowledge (e.g., the relative sizes of the bumps, their spread, and inter-bump spacings) can be easily incorporated within the Bayesian formulation.

\section{DISCUSSION}

The algorithms outlined in Section 3 only characterize the features of the inverse distribution of packet contents. Several additional issues need to be addressed before this information can be profitably incorporated into network security tools:

Relating bumps to packet groups. A feature in the inverse distribution corresponds to a set of fingerprints that are part of a content similarity group; however, this set alone does not always uniquely identify a group of packets that share content (because two groups of packets can have overlapping fingerprints).

To address this issue, we have been pursuing an approach that uses the Gaussian mixture parameters to associate with each fingerprint $f$, the probability, $p(f, i)$, that it belongs to the $i$ 'th of the $W$ components. These probabilities in turn help estimate the fraction of a packet's content that corresponds to each of the components. For a packet with fingerprint set $\left\{f_{1}, \ldots, f_{n}\right\}$, the fraction of its content that corresponds to Gaussian component $i$ is given by $x_{i}=\sum_{j=1}^{n} p\left(f_{j}, i\right)$.

Given these fractions, $\left(x_{1}, \ldots, x_{W}\right)$, one can view the packet as a point in $W$-dimensional space falling on the hyperplane that intersects each of the axes at unit distance from the origin. Intuitively, packets exhibiting content-level similarity end up getting clustered on this hyperplane. Clusters that contain higher than a threshold of their content associated with one or more non-background Gaussian components define a packet group of interest. Experiments on small-to-medium sized traces support this intuition: clusters that form are clearly separated, and each cluster does correspond to packets that exhibit content similarity.

A variety of algorithms are possible for detecting such clusters at run time, and for characterizing information about each cluster (e.g., cluster centroid, spread, exemplar packets for the cluster, etc.). Note that as new components emerge or existing ones disappear, cluster statistics need to be transformed to correspond to the new feature space that now gets defined.

Distinguishing benign and malicious packet groups. Not all packet groups that exhibit content-level similarity are malicious. In fact, such similarity may be expected in several situations, e.g., in the request and response traffic of a popular web server responding to several client requests. Two mechanisms can help reduce the likelihood of raising false alerts. First, it may be possible, as in the above situation, to identify expected sources of similar- 

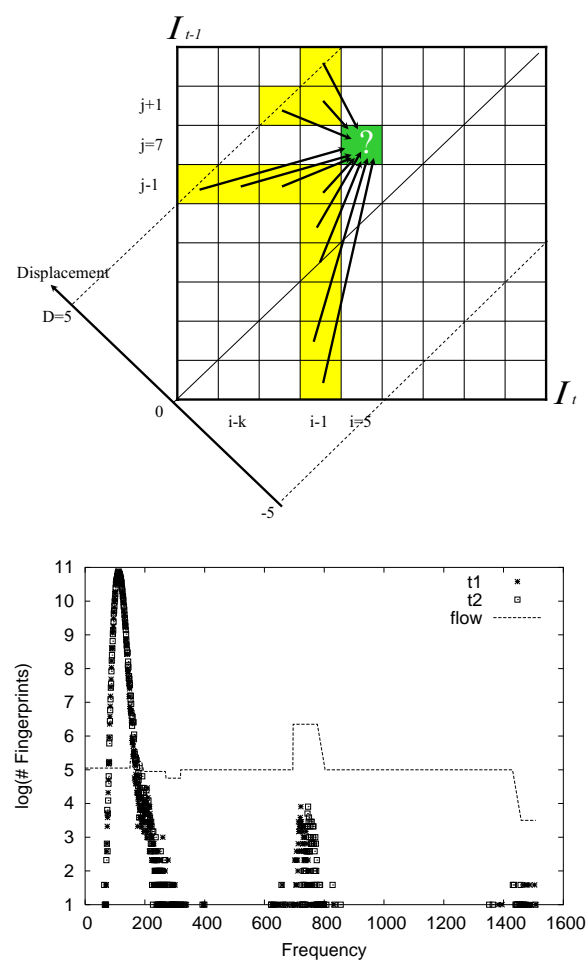

Figure 4: Dynamic programming structure (top) and the displacement field ("flow") across two epochs (bottom).

ity, which can then be simply factored out from subsequent analysis. Second, content analyses can be complemented with traditional header-based or flow-based statistics to refine the detection procedure. For example, one expects computer worm propagation patterns to show themselves as changes in the IP source and destination node connectivity structure, which would now contain a higher than expected number of edges between nodes that have previously not been big contributors to overall traffic. Such changes can be detected by extending recent stream-based traffic analyses that have identified "heavy hitter" sources and flows $[5,11,2]$. The EarlyBird system uses a simpler variant of this idea, by tracking IP source addresses that produce a large amount of traffic.

Implementation efficiency. Given the relatively heavy-weight analyses described in Section 3, one might be concerned whether such analyses can ever be used at multi-Gbps line rates. Several possibilities exist for reducing the computational cost and memory requirements of such analyses. First, cheaper header analyses can be used to filter packets for content analysis. Second, while the per-packet shingling procedure itself needs to run at line rates, the analysis of the inverse distribution can happen at larger time granularities (reflecting the aggregate impact of a group of packets). The shingling procedure itself is very regular and can benefit from a hardware assist. Third, the iterative nature of the algorithms may permit combining the iteration steps with incremental data updates.

Moreover, both the shingling step and the algorithms themselves can benefit from recent advances in sketching and sampling techniques developed for data stream analysis $[12,3]$. Recently developed stream algorithms for estimating individual points, quantiles and heavy-hitters to certain approximations [3] as well as associated communication complexity results [10] can all be extended to the inverse distribution domain. Note also that because the inverse distribution is smaller in size than its base counterpart, it lends itself to the use of more sophisticated algorithms for mixture analysis, clustering, or change detection of the kind needed by our approach.

Other uses of inverse distribution analyses on content. In addition to detecting sources of content similarity, inverse distributions of packet contents appear to have potential as compact signatures for specific (a priori known) kinds of content. For example, one can imagine such analyses being performed to detect whether copyrighted music or other media data is being transmitted out of an organization's networks. Prior work on application-level signatures based on content (eg., [14]) may also be extended to use inverse distributions.

Finally, note that although this paper has viewed packet content as a sequence of bytes, the techniques are equally applicable to other representations. This observation can enable use of inverse distribution analyses for spam detection (where content is represented as a set of keywords) and for detecting polymorphic viruses and worms (where content is represented as a set of distinguished instruction sequences).

\section{REFERENCES}

[1] A. Z. Broder, S. C. Glassman, M. S. Manasse, and G. Zweig. Syntactic clustering of the web. In Proc. WWW Conf., 1997.

[2] G. Cormode, F. Korn, S. Muthukrishnan, and D. Srivastava. Diamonds in the rough: Finding hierarchical heavy hitters in multidimensional data. In Proc. SIGMOD, 2004.

[3] M. Datar and S. Muthukrishnan. Computing rarity and similarity over data streams. In Proceedings ESA, 2002.

[4] R. Duda, P. Hart, and D. Stork. Pattern Classification. Wiley Interscience, 2nd Edition, 2000.

[5] C. Estan and G. Varghese. New directions in traffic measurement and accounting. In Proc. ACM SIGCOMM Internet Measurement Workshop, 2001.

[6] M. A. T. Figueiredo and A. K. Jain. Unsupervised learning of finite mixture models. IEEE Trans. Pattern Analysis and Machine Intelligence, 24(3):381-396, 2002.

[7] J. Jung, V. Paxson, A. W. Berger, and H. Balakrishnan. Fast portscan detection using sequential hypothesis testing. In Proc. IEEE Security and Privacy, 2004.

[8] J. O. Kephart and W. C. Arnold. Automatic extraction of computer virus signatures. In Proc. 4th Intl. Virus Bulletin Conf., 2001.

[9] H. A. Kim and B. Karp. Autograph: Toward automatic distributed worm signature detection. In Proc. USENIX Security Symp., 2004.

[10] K. Levchenko, R. Paturi, and G. Varghese. On the difficulty of scalably detecting network attacks. In Proc. ACM Symp. on Computer and Communication Security, 2004.

[11] G. Manku and R. Motwani. Approximate frequency counts over data streams. In Proc. VLDB, 2002.

[12] S. Muthukrishnan. Data stream algorithms and applications. Url : http: / / www. cs.rutgers.edu/ muthu/ stream-1-1.ps.

[13] J. Newsome, B. Karp, and D. Song. Polygraph: Automatically generating signatures for polymorphic worms. In Proc. IEEE Security and Privacy, 2005.

[14] S. Sen, O. Spatscheck, and D. Wang. Accurate, scalable in-network identification of $\mathrm{P} 2 \mathrm{P}$ traffic using application signatures. In Proc. WWW Conf., 2004.

[15] S. Singh, C. Estan, G. Varghese, and S. Savage. Automated worm fingerprinting. In Proc. OSDI, 2004. 